\title{
THE TREATMENT OF CONVERGENCE DEFICIENCY, 1933-1944*
}

\author{
BY \\ Miss Sheila Mayou \\ LONDON

\section{1.-Introduction}

THE purpose of this paper is to outline the progress made in the treatment of convergence deficiency at the Central London Ophthalmic Hospital during the past eleven years and to set out, in somewhat greater detail, the methods developed by the author which are now employed to treat it.

Patients with eyestrain have been receiving treatment in orthoptic departments for a number of years but since the war their numbers have considerably increased. Some patients can be relieved of their symptoms by the correction of their refractive errors and this may result in a permanent recovery; but if there is a true convergence deficiency, the recovery is likely only to be temporary and there may even be no improvement at all; moreover many patients complaining of eyestrain will be found to have no significant refractive errors.

Eyestrain symptoms may, of course, be due to defective muscle balance, to defective binocular vision or to uncorrected errors of refraction; these causes are not, however, covered in this paper. The cases of convergence deficiency discussed below have had any refractive errors which may have been present corrected before treatment. They have full binocular vision, little or no imbalance - not more than one or two degrees of deviation (either esophoria or exophoria) measured on the Maddox rod test : on the Maddox Wing test they may have a normal reading ( 0.01$)$ or an exophoric reading of $2 \Delta-4 \Delta$ which is said to be physiological : the majority, however, show an exophoria of $6 \Delta-8 \Delta$ and some patients show an exophoric reading of $20 \Delta$ or more. In no case which has been treated have there been any neurological or pathological conditions to which the symptoms could be attributed.

No attempt has been made to cover the treatment of the phorias -exophoria, esophoria and hyperphoria-which undoubtedly cause symptoms similar to those of convergence deficiency but are neither so frequently seen nor capable of such rapid treatment.

\footnotetext{
* Received for publication, October 13, 1945
}

All measurements referred to throughout this paper are in prism dioptres. The relationship between the prism dioptre and a degree of deviation is $7 \Delta$ prism dioptres $=4^{\circ}$ of deviation. 


\section{2.-Development of treatment}

The increasing importance of convergence deficiency cases and consequently of their treatment is, perhaps, most easily demonstrated by the following table. This is an analysis of the patients who have been seen by the orthoptic department of the Central London Ophthalmic Hospital during the years 1942-1944 classified under three headings, squints, phorias, and convergence deficiency.

TABLE I

\begin{tabular}{c|c|c|c}
\hline Year & Squints & Phorias & Convergence deficiency \\
\hline 1942 & $12+$ & 24 & 162 \\
1943 & 261 & 36 & 240 \\
1944 & 214 & 29 & 176 \\
\hline & & & \\
& 599 & 89 & 578 \\
\hline
\end{tabular}

It will be seen that over this period the number of patients suffering from convergence deficiency was approximately the same as the number of squint cases. It must be remembered, of course, that during these war years a large number of children-who normally provide something like 80 per cent. of the squint caseshad been evacuated and that until comparatively recently the L.C.C. clinics have also been closed. On the other hand, there has undoubtedly been a marked improvement in the diagnosis of convergence deficiency and consequently in the number of these cases sent for treatment.

The increased attention which has been paid to the treatment of convergence deficiency and the improvement in the results achieved is shown in the following analysis of cases treated at the Central London Ophthalmic Hospital from 1933-1944.

It will be seen from this table that of the total number of patients treated, no less than 72 per cent. were cured. The patients who showed some improvement only-7 per cent.-had all completed their training and although their tests were normal, they continued to have some symptoms. Sixteen per cent. of the patients, for one reason or another, did not complete their treatment; some of these might have been cured : most of them had shown some improvement ; but their treatment, according to the standards mentioned later in this paper, was incomplete and they are therefore not taken into account in assessing the value of the treatment. 
TABLE II

\begin{tabular}{c|c|c|c|c|c}
\hline Year & Cured & $\begin{array}{c}\text { Some } \\
\text { Improvement }\end{array}$ & $\begin{array}{c}\text { No Improve- } \\
\text { ment }\end{array}$ & $\begin{array}{c}\text { Treatment } \\
\text { Incomplete }\end{array}$ & Total \\
\hline 1933 & 1 & 1 & - & - & 2 \\
1934 & 7 & - & - & - & 7 \\
1935 & 2 & 2 & - & 1 & 5 \\
1936 & 2 & 3 & - & - & 5 \\
1937 & 3 & 1 & - & - & 4 \\
1938 & 19 & - & 1 & - & 20 \\
1939 & 3 & - & 1 & - & 4 \\
1940 & 5 & 1 & 1 & - & 7 \\
1941 & 33 & - & 2 & 7 & 42 \\
1942 & 75 & 1 & 7 & 15 & 98 \\
1943 & 110 & 12 & 3 & 21 & 146 \\
1944 & 86 & 12 & 8 & 34 & 140 \\
\hline Totals & 346 & 33 & 23 & 78 & 480 \\
\hline & & & - & & \\
\hline
\end{tabular}

The failures represent the remaining 5 per cent. and under the circumstances it may not altogether be unreasonable to assume that they were unsuitable for treatment and that their symptoms were due to their general health being at fault rather than to true convergence deficiency.

These figures are confirmed by the results obtained by Miss K. M. Parsons at Chester Royal Infirmary who employs similar methods to those in use at the Central London Ophthalmic Hospital, and by the author's records of private patients. These results are set out at Appendix " A."

A comparison between the totals in Tables I and II for the years 1942-1944 reveals an apparent discrepancy of 194 cases. The figures in Table I, however, represent all cases where the preliminary test confirmed that the patient was suffering from convergence deficiency. Table II includes only those who actually attended for 
APPENDIX "A"

1. Convergence deficiency cases seen at Chester Royal Infirmary by Miss K. M. Parsons 1942-44.

\begin{tabular}{l|r|r|r|r|r|r|r|r}
\hline & \multicolumn{3}{|c|}{ Over 30 years } & \multicolumn{3}{|c|}{ Under 30 years } & \multirow{2}{*}{ Total } & Percentage \\
\cline { 2 - 6 } & 1942 & 1943 & 1944 & 1942 & 1943 & 1944 & & \\
\hline Cured $\ldots$ & 3 & 12 & 14 & 1 & 7 & 17 & 54 & $93^{\circ} 1$ \\
Improved & 1 & 1 & - & - & - & 1 & 3 & $5 \cdot 2$ \\
No better & - & - & - & - & - & 1 & 1 & $1 \% 7$ \\
\hline & 4 & 13 & 14 & 1 & 7 & 19 & 58 & 100 \\
\hline
\end{tabular}

Note:- In addition, 14 cases were tested and were found to be suffering from convergence deficiency, but did not attend for treatment.

2. Private patients seen by Miss Sheila Mayou, 1940-44.

\begin{tabular}{l|r|r|r|r|r|r|r|r}
\hline & Under 20 & $21-30$ & $31-40$ & $41-50$ & $51-60$ & Over 60 & Total & Percentage \\
\hline Cured $\ldots$ & 15 & 22 & 18 & 8 & 12 & 6 & 81 & 87 \\
Improved & - & 1 & 3 & 1 & - & - & 5 & 54 \\
Incomplete & - & 1 & - & 2 & 1 & 1 & 5 & 54 \\
No better & - & - & 1 & - & 1 & - & 2 & 242 \\
\hline & 15 & 24 & 22 & 11 & 14 & 7 & 93 & 100 \\
\hline
\end{tabular}

treatment. The discrepancy is thus accounted for-as must be expected in a free clinic in war-time-by patients who because of the difficulties of travel or the prior claims of war-work either did not attend for treatment at all or whose attendances were too irregular for any progress to be possible.

The effects of the blitz and of the flying bombs are reflected not only in the number of attendances but also in the amount of time which it was found practicable to devote to the treatment of cases. Thus in 1940 and the early part of 1941 , work came almost to a 
standstill. In 1942 and 1943 ; the orthoptic department was working for only three half-day sessions a week : this was increased to five in September, 1943, and in February, 1944, four more were added, making a total of nine sessions a week. The advent of V1 and V2 caused a sharp falling off in attendances as many of the patients were evacuated and did not return to complete their treatment. The number of sessions was accordingly reduced, but the orthoptic department, in spite of one unpleasantly close association with a V2, was always able to handle the number of patients available.

It is worth noting that in 1942 , in spite of the war-time conditions the number of patients cured exceeded the combined total of cures obtained in the previous nine years.

The equipment in use during the latter part of the period with which this paper is concerned comprised two synoptophores, one synoptiscope and a myoscope for the treatment of patients at the rospital itself, and a diploscope and stereograms for home exercises.

\section{3.-Comparative methods of treatment}

I think most orthoptists employ methods similar to those which we use at the Central London Ophthalmic Hospital, which are described in detail later in this paper. There are, however, minor differences. It is thought that the symptoms caused by close work, which are not due to a muscle imbalance, or any refractive error, are caused by suppression. These patients can be treated by anti suppression exercises with success, and when the area of suppression has been overcome, the binocular abductions and adductions are increased to their normal range of $10 \triangle$ and $80 \triangle$. Experience has shown, that although the symptoms may have disappeared after six lessons any undue strain or illness may cause them to return. The only safe course, therefore, is to continue until all signs of suppression have gone and this usually takes twelve or more lessons.

When treating a squint, suppression is said to be overcome when diplopia is established. In the same way, when treating a convergence deficiency or an orthophoric emmetropic patient complaining of symptoms, I try to obtain diplopia, voluntary diplopia by converging the eyes, and heteronymous diplopia by looking at a distant object and seeing an intermediate object double. By making the patient aware of diplopia I do not think it necessary to spend valuable time and lessons overcoming the suppression:

An entirely different approach to the problem of convergence deficiency treatment comes from South Africa where Mr. N. A. Stutterheim treats convergence as a kinetic power of the mind 
and its insufficiency (asthenovergence) as a kinetic disorder. He maintains*:-

"Asthenovergence is the cause of eyestrain. It also plays a part in ophthalmic migraine and other disorders involving bifoveal vision. Asthenovergence can be cured by methodically calling into action the power of convergence and this is done by kinetic treatment. The power of convergence is present in every normal human brain but in cases of asthenovergence it has not been sufficiently unfolded. Kinetic treatment is certainly not a ' training' and by no means a muscle training. The ocular muscles are in no need of training in cases of eyestrain; they are strong enough without such treatment voluntarily to do many times more work than convergence asks them."'

It is not proposed in this paper to do more than touch on the methods which $\mathrm{Mr}$. Stutterheim uses which are set out in detail in his treatise. The key to his treatment, however, is the use of a battery of prisms, which are placed, base out, in front of the patient; in order to overcome the diplopia so caused, the patient is forced to converge and by gradually increasing the strength of the prisms he can be taught to maintain binocular vision over, a wide range.

On their first test, most patients complaining of eyestrain cannot overcome more than a $20 \triangle$ prism, base out, and many cannot overcome more than $10 \triangle$. Convergence power of $50 \triangle$ and over should be considered normal, although even with a fairly good range promptness and endurance may be insufficient; training is therefore usually continued until the patient is able to maintain binocular vision with an $84 \triangle$ prism base out.

Good results are claimed for these methods which require on average four to five weeks of kinetic treatment given every weekday for ten minutes, before (in Mr. Stutterheim's own words) " the power of convergence is fully unfolded."

At the Central London Ophthalmic Hospital, when the treatment of convergence deficiency first started in 1932, we worked on suppression and increased the range of convergence in order to relieve the symptoms of the few patients we had for treatment. But it soon became apparent from an examination of a number of private patients who had been treated on similar lines that after about two years the symptoms returned.

As a result, we started to teach every patient to converge voluntarily and this has remained the basis of our treatment ever since. To do this, we made the patient look at any object in front of him, for example, a small light, and taught him to converge both eyes equally without effort. This caused homonymous diplopia, which *yestrain and Convergence, by N. A. Stutterheim. Published by H. K. Lewis \&
Co., Ltd , 1937. 
he should be able to maintain for a few seconds before letting the two images merge into one. Once the patient knew what was wanted and was able to do it easily, his symptoms disappeared and it was found that very few who had been trained to this standard ever had to return for further treatment. This is the standard we now aim to achieve with all patients and we have proved conclusively that the average person, provided he has normal muscle balance and is prepared to co-operate and concentrate on his treatment, can be taught voluntary convergence in seven half-hour periods and can be freed, probably for ever, from the distressing symptoms which convergence deficiency can cause.

\section{4.- Symptoms and causes}

The most usual symptoms of which patients complain are headaches, either during or after working hours, or nausea, giddiness and occasional diplopia : other common symptoms are sore eyes and dry lids-a feeling of "grit" in the eyes, or simply a drawing feeling or acute pain in one or both eyes.

Some patients find themselves unable to read in comfort or to sew for any length of time-often as little as ten minutes being sufficient to start the symptoms; others may be unable to go to a cinema or ride in a car, bus or tram without discomfort. Some attribute the commencement of their symptoms to an illness, to overwork or to general tiredness and in nearly all cases it is found that if the patient is sent for a holiday or ceases to sew or read as the case may be, the symptoms disappear, only to become pronounced again as soon as work is resumed.

A large number of the patients who have been treated during the war have been employed on close work in artificial light. Many were women who, after years of running a home, came back either to part-time or full-time war-work and were often employed on precision work of various kinds or as clerks, typists and on telephone switchboards. Of the men, too, many were clerks, draughtsmen and machinists, working longer hours under more trying conditions than they had previously done.

Some of the most successful cases were young men, members of the A.T.C., who would later be trained as pilots for the R.A.F. or for the Fleet Air Arm under the Navy "Y" Scheme who had been turned down because of convergence deficiency. A number of these patients were treated and every one was subsequently accepted as a pilot when he had completed his treatment. A few service pilots were also seen whose eyes had become unsteady, some as a result of crashes; these, too, were all cured.

Some convergence deficiencies are of traumatic origin. In these cases it is not necessary for the patient to have been unconscious, since any severe blow to the head may be sufficient to cause the 
symptoms which can be relieved by exercises. A frontal sinus that has had surgical treatment may give similar symptoms, but these cases are usually phorias and are treated as such.

There have been a few patients with migraine. Some of these have been completely freed from further attacks as the result of treatment; others have improved in that their attacks have neither been so frequent nor so severe; few have shown no improvement. It seems clear, therefore, that a migraine patient who shows ocular symptoms can at least gain some alleviation from convergence deficiency treatment.

A type of patient who is likely to be seen more frequently in the near future is the man who has returned to civilian life after many years of active service during which his opportunities for reading and concentrating on close objects for any length of time will often have been limited. Now that he has a desk job he finds himself unable to work in comfort for more than short periods at a time.

On the other hand, the symptoms described above are not the only guide to the necessity for convergence deficiency treatment. Cases have been seen where convergence has been very poor, one eye diverging to an object held as far away as eight inches, who have not complained of any symptoms at all. These cases usually have an exophoric reading on the Maddox wing test and are sometimes referred for treatment as it is thought that their symptoms might develop in later life.

Generally speaking, patients with large exophoric readings should be given treatment whether or not they complain of symptoms, since these are liable to develop later. Patients with a low exophoric reading, however, are not treated unless symptoms are

TABLE III

\begin{tabular}{ccc|c}
\hline Age in years & Percentage of cases \\
\hline Under & 20 & $\ldots$ & $17 \cdot 7$ \\
$21-30$ & $\ldots$ & $\ldots$ & $37 \cdot 7$ \\
$31-40$ & $\ldots$ & $\ldots$ & $26 \cdot 0$ \\
$41-50$ & $\ldots$ & $\ldots$ & $10^{\circ} 7$ \\
$51-60$ & $\ldots$ & $\ldots$ & $5 \cdot 0$ \\
Over $60 \ldots$ & $\ldots$ & $1 \cdot 3$ \\
No record & $\ldots$ & $1 \cdot 7$ \\
\hline
\end{tabular}


already present; although they may sometimes be given simple exercises to practise at home by a surgeon, without being referred to an orthoptic department.

It seems clear that the age of the patient has little bearing on the question of convergence deficiency. This is shown by Table III, below, which covers all cases tested or treated at the Central London Ophthalmic Hospital during the period 1933-1944.

It will be seen that the vast majority of the patients were under forty. Perhaps too strong an inference should not be drawn from this, since it is arguable that older people have less inclination to bother with a series of attendances at a hospital. Nevertheless it is evident that convergence deficiency can be diagnosed and cured irrespective of the age of the patient.

\section{5.-The psychological approach to treatment}

This may sound a long-winded description of a simple process; but the fact remains - as is so often the case-that the patient's attitude towards treatment is all-important and may make the whole difference between success and failure. One of the essential factors in helping a patient who suffers from convergence deficiency is to explain in simple terms exactly what the trouble is and to impress upon him that a quick and permanent remedy lies largely in his own hands.

The first thing we do with a convergence deficiency is to explain exactly what the treatment entails; attendance at the Hospital for one half-hour's treatment each week, probably for six weeks, coupled with exercises at home for at least ten minutes every day. The patient is warned to expect his symptoms to be rather more pronounced during the first three treatments but is told that he may expect a definite improvement to begin after the third or fourth lesson. Most patients can find time for these lessons, particularly when they have been suffering for a long time and are really uncomfortable; the more intelligent can often manage in less than seven attendances once they understand exactly what is required of them and concentrate on their home exercises.

What many patients cannot understand is how these severe and unpleasant symptoms which may have been troubling them for many years can be overcome in so short a time and this distrust frequently makes them unwilling to go through with the treatment. In order to overcome this reluctance, we explain that although the muscles of the eyes are normal, the sight is normal and the ability to use both eyes together is normal, the power to converge or focus both eyes together on a close object is not sufficiently developed or needs too much effort; that this inability to converge or the undue effort required is the cause of the symptoms which the patient, unbeknown to himself, has been aggravating whenever 
he has drawn his eyes away from a close object instead of forcing them to look at it and maintain convergence; hence his reluctance to read or do more close work than necessary. If, however, he can be taught to converge his eyes without effort his symptoms will disappear, but that during the early stages of treatment while his eyes are learning to converge, he must expect a recurrence of the headaches and other symptoms from which he has previously suffered whenever he attempted to focus on close objects.

Anyone who tests or teaches convergence daily, as we do, is all too apt to take for granted that the patient understands what is wanted, but experience has shown that it is impossible to be too careful in explaining what is required. Many patients will look at a near object half-heartedly because it hurts them to do so or because they imagine that that is all they are asked to do. But if some definite object, such as the nib of a fountain pen, is held about six to eight inches in front of the patient's eyes and he is told " now look at the nib; make it into one object and see it clearly," he will, if he possibly can, converge both eyes in order to look at it. On being forced to converge, he will probably draw his head back from the pen nib, thus showing his dislike of looking at a close object ; it can then be explained to him that this discomfort is due to his lack of convergence, or, where he complains of diplopia, to his inability to maintain convergence. In this way he can be made aware, at the start of his treatment, of the effort which has been required whenever he has attempted to focus on any close object and he begins to have a practical understanding of the cause of his symptoms.

\section{6.-Detailed treatment}

All patients who are referred to the Central London Ophthalmic Hospital for convergence treatment, whether through an outpatient department or a private practice, have been carefully refracted. Those who have a refractive error are normally given glasses, but where the error is small, it is often better to see the result of the exercises before the glasses are ordered as they may prove to be unnecessary. In some cases, however, even after treatment, the patient may feel more rested if he wears his reading glasses.

(a) Preliminary test :-On arrival at the orthoptic department, the patient's detailed history is taken and the movements of the eyes are tested in the nine fields to see that they are normal. The visual acuity of each eye is tested and if glasses are worn constantly it is tested with and without correction. Visual acuity is usually good but sometimes one eye will read one line more than the other and occasionally amblyopic eyes are found with 6/36 vision; these amblyopic cases are more diffcult to train because, although 
their symptoms can be relieved, it is impossible to teach voluntary convergence.

The next step is to test convergence. The patient is first of all asked to look at the point of a pencil or pen held about two feet from his eyes and is told to keep looking at the point as it is brought gradually closer to his face. If he can do this until the point is within two inches of the bridge of his nose, the test can be regarded as satisfactory.

The second convergence test is to hold the pencil point about three inches from the bridge of the patient's nose and tell him to look at it; he should, with little difficulty, be able to converge both eyes and see the point as one fairly clear object.

Most patients find that they are, able to pass the first test of gradual convergence unless they have a high exophoric reading on the Maddox Wing test but a great many will, to start with, fail on the second test of jump convergence. These pencil tests can be done more accurately on the Livingston gauge which was specially designed with these tests in view.

If, however, the patient can manage both tests, he is made to look at a small object or light about six feet in front of him. By converging both eyes-by " going cross-eyed "- - he should be able to double the light and keep it double for about thirty seconds.

One of three things may happen, and it is most important that the patient's eyes are observed :-

1. Most frequently he will look at the light without any idea as to how to double it.

2. He may be able to double the light but not to maintain it for more than a moment.

3 . One eye only may converge to get the diplopia; in this case the symptoms will usually be found to be of a monocular type.

If, however, the patient can double the light by converging both eyes equally and can maintain the diplopia steadily for about thirty seconds, convergence exercises will not be necessary.

The results of the remaining tests are as follows:-On the Worth four-light test, the patient will see four lights. On the Maddox Rod test, he will usually be found to be orthophoric or to have an esophoric or exophoric deviation of not more than two degrees. On the Maddox Wing test, there may be any one of the following readings : the arrow may point to 0.01 , showing orthophoria but more usually will be between $2 \Delta-4 \Delta$ exophoria gradually moving to $8 \Delta$. Some patients, however, will see it at as much as $16 \Delta-22 \Delta$ exophoria.

On the synoptophore or synoptiscope, he may have all grades of binocular vision-simultaneous perception, fusion and stereoscopic vision; simultaneous perception slides (which have no fusion 
element) will show some suppression and his answers on the stereoscopic slides may be slow or slightly inaccurate; his fusional adduction will be below normal (few patients can adduct to $60 \triangle$ ) and he will have no adduction or voluntary convergence on simultaneous perception slides.

If the adduction is approximately $60 \Delta$ to $70 \Delta$ it may be considered good and this range of adduction or fusional convergence can be reached by gradually converging both tubes of the synoptophore until the pictures on the fusion slide break. Once they have come apart, it should be possible for the patient to make the effort of " jump convergence" and fuse the two slides together at the convergent angle. This, at first, proves to be too difficult for the average patient but comes easily with practice.

If, however, the patient can manage this, the fusion slide is replaced by a simultaneous perception slide. With the tubes set at a convergent angle of $50 \triangle$, a patient with good convergence and normal control should be able to bring the ball into the square and hold it in the centre for a few moments.

Obviously, few patients are able to do this at first and if they can, convergence exercises are unnecessary.

(b) Home Exercises:-Immediately following his preliminary test, the patient is taught how to converge and is given exercises to practise at home.

In the first of these home exercises the patient is told to hold a pen about twelve to eighteen inches from the bridge of his nose and to look at it with both eyes; he must then bring the pen closer to his nose, concentrating on it with both eyes all the time, until it is within two inches of his nose. This is similar to the gradual convergence test mentioned in para. 6(a).

When he can manage the first home exercise, the patient can go on to the second. In this, he has to hold the pen about six to eight inches from the bridge of his nose and in a direct line with a small light or torch which has been placed two or three yards away. If he looks at the light, the pen will be double due to physiological diplopia; when he looks at the pen his eyes are converged so that the light is double (homonymous diplopia). The object of this exercise is to keep the eyes in a convergent position without fusion (i.e., to converge the eyes by using the adductor muscles); the patient is therefore told to see the light double by looking at the pen and then, having removed the pen, to attempt to continue to see two lights. It is important that when the pen is removed he should continue to look at the place where it was held and not on any account look at the light. As long as the eyes stay converged, the light will remain double but immediately they are relaxed the two lights will merge into one. The patient is told to practise for about ten minutes daily throughout the period of 
treatment, until by the end of treatment he should be able to look at any object, distant or near, and without any effort, converge both eyes so as to see the object double. This is known as voluntary diplopia.

(c) Lesson one:-The patient spends only the first few minutes of this lesson on anti-suppression exercises and then goes on to fusion slides. Here, with the aid of minus lenses, he will endeavour to increase his range of convergence and at this stage it is usually best to leave him to work by himself ; provided that he really understands exactly what he is trying to do, the mere fact that he is operating the tubes himself and can feel his hands moving the slides helps him to make his eyes follow the pictures. By keeping the slides fused together, he is forcing his eyes to converge and as soon as the slides come apart he moves the tubes back until they fuse again. In this way the patient can teach himself to keep the pictures fused to $60 \Delta$ or $80 \Delta$ of conyergence.

(d) Lesson two:-The patient starts off with the exercises which he practised in lesson one, but after a few minutes with the minus lenses he should be able to converge to $70 \Delta$ with ease. The lenses are therefore removed, and he spends the rest of this lesson attempting to achieve the same standard without their aid; by the end of this lesson, most patients are able to attain $70 \triangle$ of convergence unaided, without difficulty.

(e) Lesson three :-Continuing the process of refresher training, the patient spends the first few minutes on anti-suppression exercises, followed by convergence with fusion slides. Provided he has achieved the standards set in lesson three, he then proceeds to " jump convergence" exercises. For these, the tubes are set at a convergent angle of $30 \triangle$ so that the pictures appear side by side, and by converging his eyes, the patient should be able to fuse them; when he can do this at $30 \triangle$, the convergent angle is increased to $40 \triangle$. It is important at this stage that the patient should be allowed to look away from the synoptophore between each attempt to look in and fuse the two pictures.

When the convergent angle is set above $40 \triangle$, the younger patients frequently say that the slides, when fused, appear smaller and somewhat blurred : presbyopes, on the other hand, can retain a clear image for a considerable time, varying with the degree of presbyopia.

To encourage convergence to $60 \Delta$ the patient should be told to make the pictures go out of focus and look in front of them rather than at them; this will help him to fuse the pictures together. He should be able to do this at a convergent angle of $60 \triangle$ and maintain fusion while the synoptophore tubes are locked at this angle and moved from side to side.

The next step is to teach the patient to relax his eyes and 
"break coincidence" without moving his head away from the synoptophore. Most patients find that once the pictures are fused at the convergent angle they are unable to relax sufficiently to let the fusion go so as to resolve the image into two pictures. This must be practised until it is effortless.

An alternative exercise to stimulate convergence and relaxation is to set the tubes containing the fusion slides at zero so that the patient sees the two pictures fused together; by making a voluntary effort of convergence, he should be able to break the fusion and see the pictures side by side; when he relaxes, they will fuse again. This exercise, however, is more difficult than the jump convergence exercise and should not be undertaken until the patient has mastered gradual and "jump convergence" and is making good progress with the home exercises.

During lesson three, the patient will start to use the myoscope or myoculator. This machine is not essential for the treatment of convergence deficiency but it is a great asset, particularly in a busy clinic. It consists simply of a light projected through rotating prisms on to the wall and a small motor which propels the light across the wall at a variable pace; the motor can be set to move the light horizontally, vertically, diagonally or in a circular movement.

For convergence deficiency cases, the myoscope or myoculator is used in two stages to amplify the training which the patient has done on his own in the two home exercises previously mentioned. The motor is set to produce a horizontal movement of the light at a slow pace and for the first stage the patient is told to hold his finger close to his face and keep his finger in line with the light as it travels across the wall. By looking at his finger, he will see the projected light double and should try to maintain the two lights over the whole range of movement. When he can do this, the patient goes on to the second stage in which he attempts to achieve exactly the same result-the doubling of the light-but without using his finger to help him, by keeping his eyes converged to the arc where the finger was travelling.

Almost invariably, when patients first use the myoscope they try to move their heads or their bodies instead of their eyes and as soon as the finger is removed they focus on the light which becomes single; this tendency needs careful checking; only regular practise at the home exercises and sufficient encouragement during this and the succeeding lessons will enable the patient to master this exercise; but although, at times, he may feel that he will never be able to manage it, success will come suddenly.

(f) Lesson four:-A quick run-through the exercises of the previous lessons usually reveals that further time must be spent on " jump convergence," relaxation, converging from zero and the myoscope exercises; these are accordingly continued. 
When, however, they have been mastered, the patient goes on to the voluntary convergence exercise. For this, the fusion slides in the synoptophore are replaced by simultaneous perception slides. These are slides which have no fusion element but have two dissimilar images such as a ball and a square. If the patient has good convergence and is not relying on his fusion power to hold it, he will, by converging both eyes, be able to pull the ball into the square at a convergent angle between $10^{\circ}$ and $35^{\circ}$. At first, he may be able to move the ball towards the square or even put it in but will not have the power to keep it steadily in the centre for any length of time. This, however, comes quickly with practice.

By the end of this lesson, the patient should also be able to follow the myoscope light in all directions, making it appear double or single at will, without effort.

By now, too, he should be able to converge or relax on a stereoscope card without the use of the stereoscope, so that he can see the different formations of the letters if the card is simply held up in front of him. This is a simple exercise and should also be continued at home. All that is required is a postcard with two letters, $\mathrm{F}$ and $\mathrm{L}$, about $\frac{1}{2} \mathrm{in}$. in height and the same distance apart ; alternatively, any simple stereoscope card can be used. To relax, the patient first looks at the card and sees $\mathrm{F}$ and $\mathrm{L}$; then, by looking right through the card into the distance, his eyes are slightly diverged from the card and he sees the letters as FEL. To converge, he fuses a near object held between his eyes and the card and as his eyes are too convergent to the card he once again sees the letters as FEL; the near object is then removed and the patient should be able to maintain the convergence, until eventually he should be able to converge without the help of the near object. This converging and relaxing-FEL relaxed, FL normal, and FEL converged-helps to give a general tone up to the abductor and adductor muscles.

This exercise can also be practised on the diploscope. The patient will aim to change from the normal DOG in three holes to DOOG in four holes relaxed and to OGDO in four holes converged and should be able to manage each position without difficulty by the time treatment is complete. The diploscope is, however, to some extent a luxury with hospital patients and it is only used in the clinic and not for home exercises.

(g) Lesson five :- This is spent on a general work-through of all the exercises which the patient has learned. Some patients will not yet have achieved the necessary standard on one or more of the exercises and may not have covered the full syllabus of the previous lessons; others will spend this lesson on a general tone up; a few will be able to dispense with further exercises altogether. The 
average patient will still need some further treatment, however, and particular attention is paid during this lesson to the voluntary convergence exercises since they contribute most of all to accuracy of convergence.

(h) Final test:-In the preliminary test, a careful enquiry was made into the symptoms noted in each case and it is usually found that these symptoms disappeared as soon as voluntary convergence was established.

The patient is now ready for the final test which comprises a series of routine checks. Convergence should by now be effortless and voluntary convergence should enable the patient to double any small image which is at least a yard away and to hold the two images for a short time without difficulty. The test for visual acuity will show improved vision-possibly as much as a line better -due to the fact that suppression has been overcome even though comparatively little time has been spent on anti-suppression exercises. The Worth four lights are seen as four unchanged; the Maddox Rod measurement is usually orthophoric or shows exophoric or esophoric deviation of $1 \Delta$ which has probably remained unchanged. There may be little or no alteration in the deviation on the Maddox Wing test; if the patient had an exophoria of $4^{\Delta}-6 \Delta$ before treatment, this will probably measure exophoria $2 \Delta-4 \Delta$ or may be unchanged after treatment. If, however; his deviation was $14 \Delta-16 \Delta$, this may vary after treatment anywhere between $2^{\circ}-16^{\circ}$; these different results do not, however, seem to matter, and some patients do come down to normal. Synoptophore tests should show no deviation and little or no suppression with simultaneous perception slides. Voluntary convergence with simultaneous perception slides should be good, as should gradual and " jump convergence" to $80 \triangle$ with relaxation; lastly, the patient should have good abduction power to $10 \triangle$ and full stereoscopic vision.

Provided he can pass all these tests and is free from symptoms, the patient is ready for discharge. Careful supervision is necessary, however, throughout the period of treatment as a few cases become esophoric and may start convergent spasms which are difficult to overcome. This should not occur if sufficient attention is paid to relaxation exercises and it is therefore essential that patients suffering from convergence deficiency should be under constant supervision and that, once convergence is established, part of each lesson should be spent on relaxation.

\section{7.-Conclusions}

From the author's experience of convergence deficiency cases over the past eleven years, it is suggested that the following general conclusions may be drawn :- 
(a) The number of patients of all ages suffering from convergence deficiency is large and the symptoms, which may include migraine, extremely unpleasant; the psychological element is often present and particular attention must be paid to the patient's mental approach both before and during treatment since the successful remedy lies largely in his own hands.

(b) Care should be taken when testing and treating a patient with an esophoria since many of these have a convergence deficiency as well.

(c) Even patients who are orthophoric and can actually comverge may benefit from treatment, while many patients with small refractive errors are able to dispense with their correction after they have been treated.

(d) Above all, treatment can be quick and effective-on average, two tests and five half-hour lessons-but must be taken under trained supervision; it should aim at building up voluntary convergence and teaching the patient to relax once convergence is established, since voluntary convergence alone can prevent a recurrence of the symptoms in after years. If a patient is discharged as cured after undergoing the treatment described in this paper, it may be said with some certainty that with the exception of the patient with migraine symptoms who may need a subsequent refresher course, the risk of his having to return for further treatment is infinitesimal.

\section{THE ULTRA-VIOLET OPHTHALMOSCOPE*}

BY

\section{J. De Ment \\ OREGON, U.S A.}

THE ophthalmoscope has long been used for observing the interior of the eye, and thus determining the appearance of the media, the condition of the retina, choroid, and optic nerve, and for a variety of other conditions of this organ. As is well known, the presentday ophthalmoscope relies upon the use of white light and ordinary vision, though in past years a number of modifications have been introduced into the design and mode of operation of this instrument.

The fluorescence of the eyes of humans and lower organisms has been known since the earliest days of fluoro-chemistry, though the first accurate description of this phenomenon was probably

\footnotetext{
* Received for publication, November $4,1944$.
} 